\title{
Therapeutic evaluation of local drug delivery system containing $1.5 \%$ chlorhexidine used as an adjunct to scaling and root planing for the treatment of chronic periodontitis - A clinical and microbiologcal study
}

\author{
Randhir Kumar ${ }^{1}$, Geeta Sharma ${ }^{2 *}$ \\ 1,2Professor and HOD, ${ }^{1}$ Dept. of Periodontology, ${ }^{2}$ Dept. of Oral \& Maxillofacial Pathology, ${ }^{1}$ Patna Dental College Patna, Bihar, ${ }^{2}$ Sarjug
} Dental College, Darbhanga, Bihar, India

*Corresponding Author: Geeta Sharma

Email: docgeetarandhir@gmail.com

\begin{abstract}
Periodontitis has a multifactorial etiology with primary etiologic agents being pathogenic bacteria that reside in the subgingival area. Local delivery devices are systems designed to deliver agents locally into periodontal pocket to retain therapeutic levels for a prolonged period of time.

Review of Literature: Goodson et al $(1979)^{1}$ introduced the use of tetracycline as a local drug delivery component in the treatment of periodontal diseases. W.A. Soskolne, P.A. Heasman, and H.N. Newman $(1997)^{5}$ conducted a multicenter study to evaluate the safety and efficacy of a degradable, subgingivally placed drug delivery system containing $2.5 \mathrm{mg}$ Chlorhexidine were evaluated in a randomized, blinded, multi-center study.

Aims and Objective: The aims of the present study is to compare the effect of experimental local drug delivery system containing $1.5 \%$ chlorhexidine (chlorhexidine digluconate $0.5 \%$ and chlorhexidine dihydrochloride $1 \%$ ) with scaling and root planing and scaling and root planing alone by assessing the effect on: Plaque, Gingival Inflammation, Bleeding on probing, Pocket depth, Relative attachment levels, Percentage of spirochetes, motile rods and coccoid cells.

Materials and Methods: A total of 30 subjects comprising of both the sematerxes and diagnosed as suffering from chronic localized or generalized periodontitis.

Recording of Clinical and Microbiological Parameters: Plaque index, gingival index, Pocket depth measurement using William's graduated probe using acrylic stent.

Microbiological study of collected plaque sample for coccoids, rods and spirochetes under dark field microscopy. The above clinical and microbiological parameters were recorded on: 0 day, $30^{\text {th }}$ day, $45^{\text {th }}$ day.

Statistical Analysis: Post treatment changes from baseline to different time intervals in various clinical parameters were analyzed by paired t-test (Intragroup). Intergroup comparisons of post-treatment changes were analyzed by unpaired t-test. p-value $<0.05$ was considered significant difference.

Results and Discussion: At the selected sites clinical and microbiological parameters were recorded at base line, $30^{\text {th }}$ day and $45^{\text {th }}$ day, as per the study design. The experimental material along with scaling and root planing is found to be more effective than scaling and root planing alone when evaluated clinically and microbiologically. This effect may be due to antimicrobial and anti-plaque property of the experimental material.
\end{abstract}

Keywords: Periodontitis, Local drug delivery system, Chlorhexidine scaling.

\section{Introduction}

Periodontal disease is a chronic inflammatory destructive disease affecting one third of the adult population. Periodontitis describes a group of related inflammatory diseases resulting in destruction of the tissues that support the tooth. Periodontitis has a multifactorial etiology with primary etiologic agents being pathogenic bacteria that reside in the subgingival area. The goal of periodontal therapy is to prevent, arrest, control or eliminate periodontitis and to restore the lost form, function, esthetics and comfort. Periodontal therapy has been directed at altering the periodontal environment to one, which is less conducive to the retention of bacterial plaque in the vicinity of gingival tissue.

Local delivery devices are systems designed to deliver agents locally into periodontal pocket to retain therapeutic levels for a prolonged period of time. Controlled release local delivery systems, in which the antimicrobial is available at therapeutic levels for several days, have been evaluated in several forms and using different antimicrobials. Different drugs used for local delivery are tetracyclines including doxycycline and minocycline, metronidazole and chlorhexidine. These devices are less invasive treatment options and it requires less time compared to surgical treatment. Chlorhexidine is one of the most effective topical agents, long been used as an effective antimicrobial agent. Short-term use of chlorhexidine causes a striking reduction in the number of oral microorganisms. In the present study an attempt has been made to evaluate and compare the efficacy of gel containing chlorhexidine digluconate and chlorhexidine dihydrochloride having $1.5 \%$ of chlorhexidine as an adjunct to scaling and root planning.

\section{Review of Literature}

The concept of treating periodontitis by providing chemotherapeutic agents in that site by specific controlled release delivery systems has also undergone considerable development over the last two decades. Pioneering work by Goodson et al (1979) $)^{1}$ introduced the use of tetracycline as a local drug delivery component in the treatment of 
periodontal diseases. The group demonstrated that treatment could alter the periodontal microflora and reduced gingival inflammation. W.A. Soskolne, P.A. Heasman, and H.N. Newman $(1997)^{5}$ conducted a multicenter study to evaluate the safety and efficacy of a degradable, subgingivally placed drug delivery system containing $2.5 \mathrm{mg}$ Chlorhexidine were evaluated in a randomized, blinded, multi-center study. An analysis of patients with initial probing depths of 7 to $8 \mathrm{~mm}$ revealed a significantly greater reduction in PD and CAL in those pockets treated with chlorhexidine compared to SRP at both 3 and 6 months. Soskolne WA, Chajek T, Flashner M, et. al. $(1998)^{6}$ evaluated the release profile of Chlorhexidine from the PerioChip, a biodegradable local delivery system that contains $2.5 \mathrm{mg}$ of Chlorhexidine gluconate in a cross-linked hydrolyzed gelatin matrix, into the gingival crevice. These results indicate that the PerioChip can maintain clinically effective levels of chlorhexidine in the GCF of periodontal pockets for over 1 week without any detectable systemic absorption. Negih Azmak, Gul Atilla, Hanne Luoto and Timo Sorsa ${ }^{7}$ evaluated the efficacy of subgingival controlled release delivery of Chlorhexidine chip on clinical parameters and MMP-8 levels in gingival crevicular fluid in chronic periodontitis patient. Results of their study showed the significant improvement in probing depth and clinical attachment level when compared the chlorhexidine and SRP group with SRP alone at 3 and 6 month.Intergroup analysis demonstrated significantly lower mean levels of gingival crevicular fluid MMP-8 level for the chlorhexidine and SRP group with SRP alone group. Cosyn J, Wyn I, De Rouck T, Moradi Sabzevar M.A. ${ }^{9}$ investigated the clinical outcome of a subgingivally applied Chlorhexidine varnish when used as an adjunct to scaling and root planing in the treatment of chronic periodontitis. A randomized controlled, single blind, parallel trial was conducted. The finding of this study showed that additional reduction of probing pocket depth and gain of attachment in sites where scaling and root planing with application of Chlorhexidine varnish compared with scaling and root planing alone.

\section{Aims and Objective}

The aims of the present study is to compare the effect of experimental local drug delivery system containing $1.5 \%$ chlorhexidine (chlorhexidine digluconate $0.5 \%$ and chlorhexidine dihydrochloride $1 \%$ ) with scaling and root planing and scaling and root planing alone by assessing the effect on: Plaque, Gingival Inflammation, Bleeding on probing, Pocket depth, Relative attachment levels, Percentage of spirochetes, motile rods and coccoid cells. The present study is carried out with an objective to provide a local drug delivery system which is expected to play a role as an anti-microbial, anti-inflammatory and anti-plaque agent and is also biologically acceptable, easy to apply, require less chair side time, and cost-effective.

\section{Materials and Methods}

A total of 30 subjects comprising of both the sexes and diagnosed as suffering from chronic localized or generalized periodontitis, above 20 years of age were considered for the present study. All potential participants were explained the need and design of the study. After recording of preliminary information, acrylic stent was prepared. Selected sites were randomly divided into control sites and experimental sites, which were treated by using split mouth design:

\section{Recording of clinical and microbiological parameters}

Plaque index, Gingival index, Pocket depth measurement using William's graduated probe using acrylic stent, Relative distance between base of pocket and fixed reference point on the stent for assessing clinical attachment gain or loss;, Microbiological study of collected plaque sample for coccoids, rods and spirochetes under dark field microscopy. The above clinical and microbiological parameters were recorded on: 0 day, $30^{\text {th }}$ day, $45^{\text {th }}$ day.

\section{Statistical analysis}

Post treatment changes from baseline to different time intervals in various clinical parameters were analyzed by paired t-test (Intragroup). Intergroup comparisons of posttreatment changes were analyzed by unpaired t-test. p-value $<0.05$ was considered significant difference.

\section{Results and Discussion}

This study comprised of 30 subjects at the completion of the study. The subjects were selected on the basis of pocket depth of 5-7 $\mathrm{mm}$ in different quadrants of the mouth using a split mouth design. The selected sites were randomly grouped as control and experimental sites. Control sites were treated with Scaling and root planing and the experimental sites were treated with Scaling and root planing followed by placement of experimental local drug delivery system. At the selected sites clinical and microbiological parameters were recorded at base line, $30^{\text {th }}$ day and $45^{\text {th }}$ day, as per the study design. The experimental material along with scaling and root planing is found to be more effective than scaling and root planing alone when evaluated clinically and microbiologically. This effect may be due to antimicrobial and anti-plaque property of the experimental material.

It is clear from the results of present study that this combination along with scaling and root planing is effective in reducing dental plaque level, reducing gingival inflammation, reducing pocket depth and also results in gain in clinical attachment. It also controls the localized infection and prevents new lesion formation.

The local drug delivery system used in the present study is simple and easy to use. Its syringeability allows easy insertion into the pocket also it can reach into depth of tortuous pocket due to its gel consistency. The drug being bioadhesive as well as the property of becoming thicker after coming in contact with gingival cervicular fluid allow better retentivity in the pocket. It is also biologically accepted without any side effects and was well tolerated by the subjects. 
Graph 1: Change in plaque index comparison between control and experimental sites

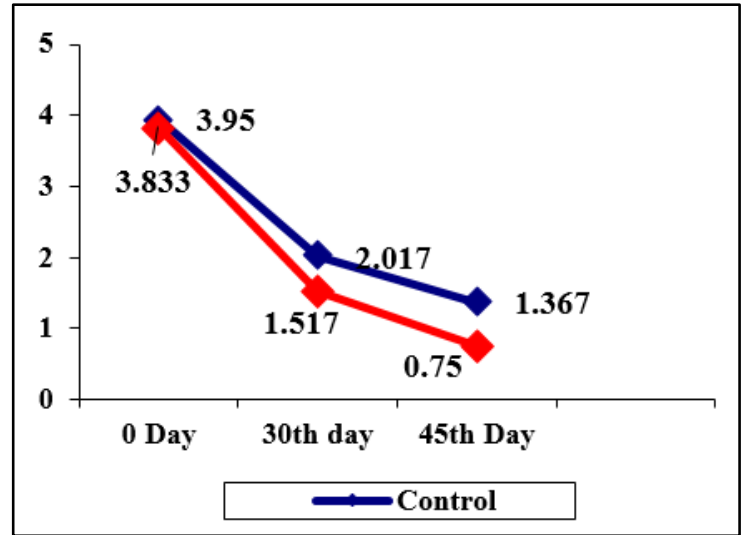

Graph 2: Change in Gingival Index Score Comparison between control and experimental sites

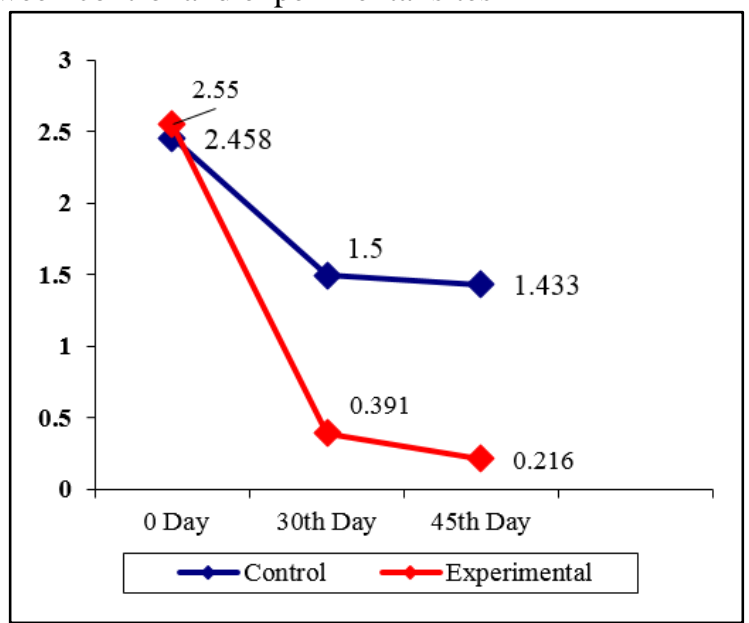

Graph 3: Change in probing pocket depth comparison between control and experimental sites

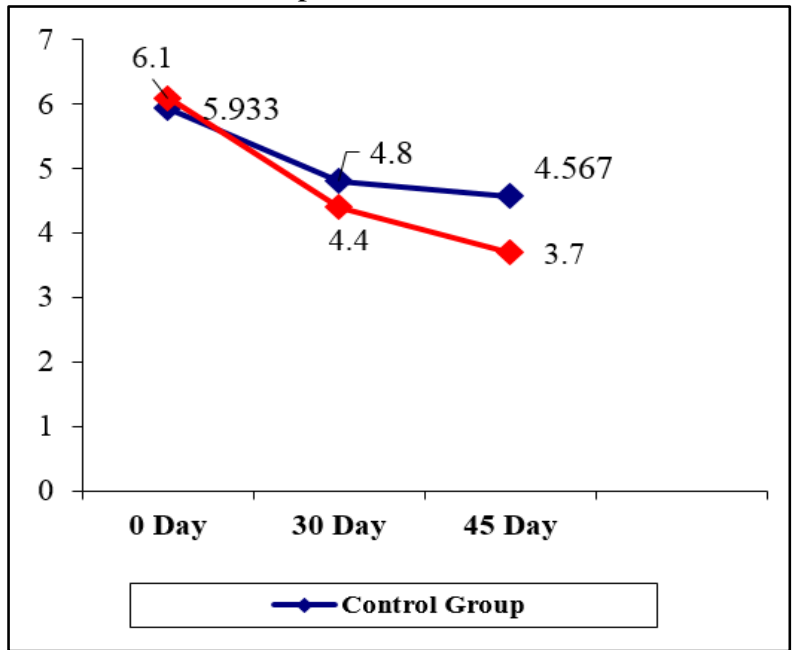

Graph 4: Change in relative attachment levels comparison between control and experimental sites

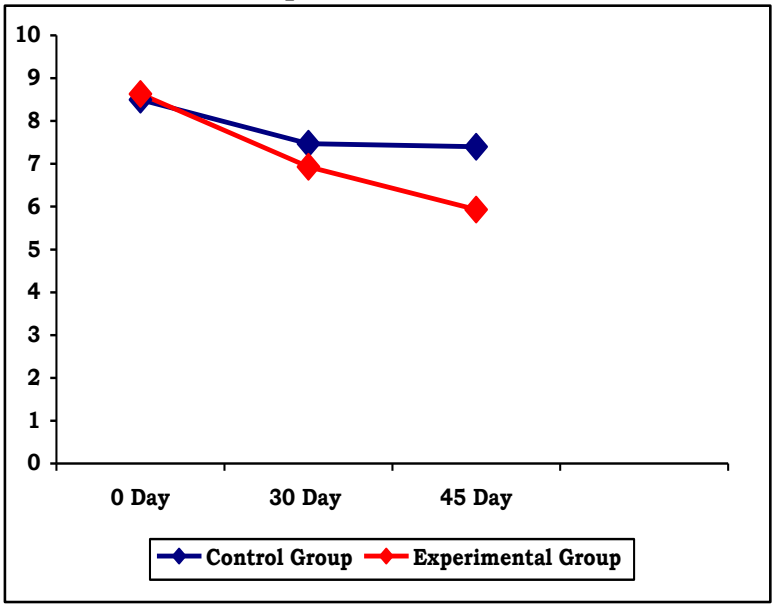

\section{Source of funding}

None.

\section{Conflict of interest}

None.

\section{References}

1. Goodson JM, Haffajee AD, and Socransky SS. Periodontal therapy by local delivery of tetracycline. J Clin Periodontol 1979;6:83-92.

2. Lindhe J, Heijl L, Goodson JM. Local tetracycline delivery using hollow fiber devices in the periodontal therapy. J Clin Periodontol 1979;6:141-9.

3. Noguchi T, Izumizawa K, Fukuda M, Suzuki Y, Kitamura S and Ikura $\mathrm{H}$. New method for local drug delivery using resorbable base material in periodontal therapy. Bull Tokyo Med Dent Uinv 1984;31:145-53.

4. Oosterwaal PJM, Mikx FHM, van Hof MA, Renggli HH. Comparison of the antimicrobial effect of the application of chlorhexidine gel, amine fluoride gel, and stannous fluoride gel in debrided periodontal pockets. J Clin Periodontol 1991;18:245-51.

5. Soskolne WA, Heasman PA, Stabholz. Sustained local delivery of chlorhexidine in the treatment of periodontitis: a multi-center study. J Periodontol 1997;68:32-8.

6. Soskolne WA, Chajek T, Flashner M. An in-vivo study of the chlorhexidine release profile of the PerioChip in the gingival crevicular fluid, plasma and urine. J.Clin. Periodontol. 1998;25:1017-1021.

7. Negih Azmak, Gul Atilla, Hanne Luoto and Timo Sorsa. The effect of subgingival controlled-release delivery of chlorhexidine chip on clinical parameters and matrix metalloproteinase-8 levels in gingival crevicular fluid. $J$ Periodontol 2002;73:608-15.

8. Cosyn J, Wyn I, De Rouck T, Moradi Sabzevar M.A. A chlorhexidine varnish implemented treatment strategy for chronic oeriodontitis. J Clin Periodontol 2005;32:750-6.

9. Stabholz. A, Shapira L, Mahler D. Role of PerioChip in treating adult periodontitis: an interim report. Compend Contin Educ Dent 2000;21:325-8.

10. Adriana Helena Chikaro, Luciene Cristiana de, Elcio Marcantonio et al. Subgingival utilization of $1 \%$ chlorhexidine collagen gel for the treatment of periodontal pockets. A clinical and microbiological study. Braz Dent J 2001;12:209-13. 
11. Perinetti G, Paolantonio M, Cordella C, D'Ercole S, Serra E, Piccolomini R. Clinical and microbiological effects of subgingival administration of two active gels on persistent pockets of chronic periodontitis patients. J Clin Periodontol 2004;31:273-81.

12. Adriana Helena Chikaro, Luciene Cristiana de, Elcio Marcantonio. Subgingival utilization of $1 \%$ chlorhexidine collagen gel for the treatment of periodontal pockets. A clinical and microbiological study. Braz Dent J 2001;12:209-13.

How to cite this article: Kumar R, Sharma G. Therapeutic evaluation of local drug delivery system containing $1.5 \%$ chlorhexidine used as an adjunct to scaling and root planing for the treatment of chronic periodontitis - A clinical and microbiologcal study. Int $J$ Periodontol Implantol 2019;4(3):103-6. 